\title{
CONSIDERACIONES SOBRE EL HIDROCEFALO DE BAJA PRESION
}

\author{
E. Herskovits ** \\ R. MATERA JR. * \\ R. MAtera***
}

El sindrome de hidrocéfalo de baja presión o de presión normal obtuvo, en los últimos años, real ubicación en la inquietud de $\operatorname{los}$ médicos $1,4,9,10,11$. También ha abierto una expectativa sobre un cuadro demencial cuya característica primordial es su reversibilidad ${ }^{14}$.

El sindrome clínico de hidrocéfalo con presión normal de líquido cefalorraquídeo tiene como sintomas fundamentales cambios mentales y alteraciones en la marcha. Generalmente los trastornos mentales son los primeros en aparecer, aunque esto no es invariable. La iniciación de la enfermedad es solapada; disminuye la espontaneidad, se aprecia una apatía y luego comienzan los olvidos para los hechos recientes. Paulatinamente se observa una disminución en los procesos mentales del paciente, con trastornos de la palavra y de la escritura, del dibujo y de la copia. Los trastornos en la marcha se caracterizan por un enlentecimiento y pasos inseguros, que han sido dudosamente definidos como apraxia de la marcha; se observa hiperreflexia, ocasionalmente reflejos de prensión forzada, chupeteo ,incontinencia urinaria y nistagmus $4,6,23,29$. Se ha relacionado este sindrome a la existencia previa de causas capaces de modificar, en alguna forma, la dinámica del líquido cefalorraquídeo: traumatismos, hemorragias cerebromeningeas, infecciones, tumores $\mathrm{y}$ malformaciones ${ }^{2,7,9,17,25}$.

Interpretación fisio-patológica - En el año 1964 Hakim ${ }^{10}$ describió el sindrome clínico de hidrocefalia con presión de líquido cefalorraquídeo normal, y dió su interpretación fisio-patológica según la cual dicho sindrome se produciría por el desequilibrio hidrodinámico entre la presión del líquido cefalorraquídeo y la presión del sistema vascular. Las venas intracraneanas actúan como un sistema abierto, ya que su volumne sanguíneo varía a través de un libre intercambio con la sangre venosa sistémica. El líquido cefalorraquídeo actúa como un sistema cerrado, debido a su circulación restringida y unidireccional hacia las vellosidades aracnoideas, y está abierto a la sangre venosa sistémica sólo a través del sistema venoso intracraneano.

Debido a la naturaleza visco-elástica del tejido cerebral, la presión del líquido cefalorraquídeo no se transmite uniformemente hacia el sistema venoso

Centro de Investigaciones Neurológicas, Buenos Aires, Argentina: * Médico residente, Neurologia; ** Professor Auxillar de Clínica Neurológica U.N.B.A.; *** Proiessor Titular de Neurocirugía U.N.B.A. 
del parénquima cerebral. La acción mecánica de cada sistema a través de dícho parénquima, no se hace por presión, sino por transmisión de líneas de fuerza. La fuerza efectiva de la expansión dentro de los ventrículos no es equivalente sólo a la presión ventricular, sino que es producto de la superficie o área ventricular y la presión. Asímismo, la fuerza opuesta ejercida por el sistema venoso es producto del promedio de la presión venosa y el área efectiva de la venas dentro del parénquima cerebral.

El efecto de estas dos fuerzas opuestas sobre el tejido cerebral no depende de la suma de las fuerzas que estos dos sistemas ejercen sobre el cerebro, sino más bien su efecto se debe al desbalance entre ellas. Si están desbalanceadas, el movimiento resultante produce un campo de distorsión y esfuerzo en algunas regiones del tejido cerebral, que inducen a una ingurgitación o compresión del sistema venoso. Por este mecanismo, el balance entre las dos fuerzas puede mantenerse con cualquier valor de la presión del líquido cefalorraquídeo.

El aumento del volumen ventricular produce un colapso progresivo de las venas, especialmente de aquellas que están más cerca de esta cavidad; este colapso reduce la circulación con la consiguiente hipoxia que trae aparejado cambios bioquímicos $\mathrm{y}$ metabólicos con pérdida de lípidos y proteínas $\mathrm{y}$, por supuesto, atrofia.

Las líneas de esfuerzo son concéntricas y principian alrededor de los ventrículos, propagándose centrifugamente hacia la superficie del cerebro, de tal forma que su corteza es la última estructura que se complica 5, 6, 8, 19, 24 . Estas líneas de fuerza también son transmitidas al tronco cerebral, y es posible que en hidrocéfalos con presión normal o alta, los desórdenes de la marcha atribuidos a una distensión de la fibras superiores del tracto piramidal sean también debidas a distorsión del mesencéfalo ${ }^{27}$.

Hemos visto que, cuando por cualquier causa aumenta la presión del líquido cefalorraquídeo en primera instancia, el tejido cerebral es, por lo tanto, desplazado debido al desbalance entre las dos fuerzas. El sistema venoso se colapsa, y esta situación continuará durante el tiempo que la presión del liquido cefalorraquídeo permanezca alta; los ventrículos se agrandan en volumen, tanto como el sistema venoso cede en la misma forma, lo cual corresponde a la fase mecánica del agrandamiento ventricular. La propagación de este desbalance produce con el tiempo pérdidas de proteinas y lípidos y esto permite un empeoramiento en el desbalance, que corresponde a la fase metabólica o química del hidrocéfalo. Si en este momento suprimimos la causa de la hipertensión del líquido cefalorraquideo, el sistema igual continuará desbalanceado por el aumento del área o superficie ventricular que, aún con presión normal del líquido cefalorraquídeo, mantiene el desbalance si suponemos que la fuerza es igual a la presión por su superficie de acción.

Reduciendo la presión del líquido cefalorraquídeo por debajo del valor normal, se encuentra un punto en que la fuerza efectiva del sistema del 
líquido es menor que la del venoso. Como consecuencia el tejido cerebral se puede mover de nuevo a su posición inicial disminuyendo el área ventricular; en este momento se aumentará la presión del líquido cefalorraquideo a su valor inicial, y el equilibrio persistirá. Esto se conseguiría mediante la implantación del sistema valvular que funciona con presión inferior a la normal del enfermo ${ }^{22,20,28}$. No es la presión del líquido cefalorraquídeo lo que determina el tamaño de los ventrículos, sino la diferencia de las fuerzas entre los dos sistemas.

Es importante diferenciar con fines prácticos el agrandamiento del ventrículo como consecuencia de un desbalance hidrodinámico (hidrocéfalo verdadero) y el de los ventrículos como resultado de una atrofia (hidrocéfalo exvacuo), según conceptos de Hakim, pues en ello estriba la diferencia terapéutica y pronóstica. Sin embargo, esta teoría de Hakim sobre la fisiopatogenia del hidrocéfalo de presión normal, ha encontrado objeciones entre las que cabe mencionar el trabajo de $O$. Barini ${ }^{3}$, quien hace una revisión exhaustiva sobre los mecanismos físicos que diversos autores han considerado sobre el particular.

Mientras que el hidrocéfalo de presión normal implicaria un proceso reversible, la atrofia es irreversible, y el resultado es la pérdida de tejido cerebral. Dentro de estos procesos se incluyen el envejecimiento, los cambios metabólicos, reacciones enzimáticas, infecciones virales, enfermedades de Alzheimer, Pick, Jacob-Creutzfeldt, corea de Huntington, parálisis general de origen sifilítico.

Métodos de diagnóstico - Comentaremos únicamente aquellos estudios complementarios que son de valor para el diagnóstico de hidrocéfalo con presión de líquido cefalorraquídeo normal.

Neumoencefalografía - Demuestra un agrandamiento del sistema ventricular, especialmente en los cuernos frontales y por poco o ningún aire en los espacios subaracnoideos, por arriba de las cisternas basales. El ángulo del cuerpo calloso, en términos generales, se encuentra próximo a los $120^{\circ} 16,26$. Algunos enfermos, despuées de la neumoencefalografía pueden empeorar sus sintomas.

Punción lumbar - Una de las condiciones fundamentales para afirmar este sindrome es que la tensión del líquido cefalorraquideo, en decúbito lateral, no sobrepase los $16 \mathrm{~cm}$. de agua. El examen citoquímico del líquido cefalorraquídeo debe ser normal. La posibilidad que la tensión del líquido se encuentre elevada en presencia de este sindrome debe ser tomada en cuenta para los primeros estadios de la enfermedad, aunque debiera descartarse completamente esta eventualidad, por el hecho de que, con una tensión de líquido cefalorraquídeo elevada, no hay sintomas característicos de esta enfermedad. Se ha descripto que la extracción del líquido cefalorraquídeo en cantidad de 10 a $20 \mathrm{~cm}$ diariamente, produce notoria mejoría en algunos de los paciente, al menos por algunas horas; sin embargo, dada la subjetividad de este método, no nos parece del todo fiable. 
Cisternografía isotópica - Este método revela actividad ventricular persistente por más de 24 a 48 horas, con poca o ninguna captación en corteza ${ }^{13},{ }^{18}$. En nuestros enfermos se realizó también electroencefalograma, cuyo patrón fué una desorganización generalizada, inespecífica.

\section{CASUISTICA}

Durante el transcurso de 3 años hemos podido seleccionar y operar 10 pacientes que configuraban un sindrome de hidrocéfalo de baja presión tanto de un punto de vista clínico como por sus exámenes complementarios. Con el objeto de facilitar la comprensión de nuestros casos, realizamos los cuadros demostrativos de los hechos más prominentes.

\begin{tabular}{|c|c|c|c|}
\hline Caso & Sexo & Etiologia & $\begin{array}{l}\text { Tiempo de } \\
\text { evolución }\end{array}$ \\
\hline 1 & $\mathbf{M}$ & Traumatismo & 24 meses \\
\hline 2 & $\mathbf{M}$ & Hemorragia cerebromeningea & 3 meses \\
\hline 3 & $\mathbf{M}$ & Traumatismo & 10 meses \\
\hline 4 & $\mathbf{M}$ & Tumor cerebral benigno & 10 meses \\
\hline 5 & $\mathbf{M}$ & Traumatismo & 26 meses \\
\hline 6 & $\mathbf{M}$ & Traumatismo & 4 meses \\
\hline 7 & $\mathbf{M}$ & Traumatismo & 6 meses \\
\hline 8 & $\mathbf{M}$ & Traumatismo & 18 meses \\
\hline 9 & $\mathbf{M}$ & Traumatismo & 20 meses \\
\hline 10 & $\mathbf{F}$ & Trombosis & 6 meses \\
\hline
\end{tabular}

Tabla 1 - Sexo, etiología y tiempo de evolución en los 10 casos,

\begin{tabular}{|c|c|c|c|}
\hline Caso & Operación & Válvula & Resultados \\
\hline 1 & V.H. & L.L. & favorable * \\
\hline 2 & V.P. & L. & favorable * \\
\hline $\mathbf{3}$ & V.P. & L. & sin cambios \\
\hline 4 & V.P. & L. & favorable * \\
\hline 5 & V.H. & L.L. & favorable * \\
\hline 6 & V.H. & L.L. & fallecido \\
\hline 7 & V.H. & L.L. & favorable * \\
\hline 8 & V.H. & L.L. & sin cambios \\
\hline 9 & V.H. & L.L. & sin cambios \\
\hline 10 & V.H. & L.L. & favorable * \\
\hline
\end{tabular}

Tabla 2-Resultados terapéuticos: V.P. = válvula de Pudens; V.H. = válvula de Holter; L. = low; L.L. - low low; * favorable = mejorfa de un $50 \%$ de los sintomas prominentes. 


\section{COMENTARIOS}

Nuestros 10 enfermos presentaron las características clínicas descriptas en este sindrome, y un patrón común en todos los estudios complementarios realizados (neumoencefalografía, cisternografía radioisotópica y examen del líquido cefalorraquídeo). Debemos mencionar asímismo que estos 10 enfermos fueron seleccionados de un total de 21 enfermos que presentaban las características clínicas del sindrome.

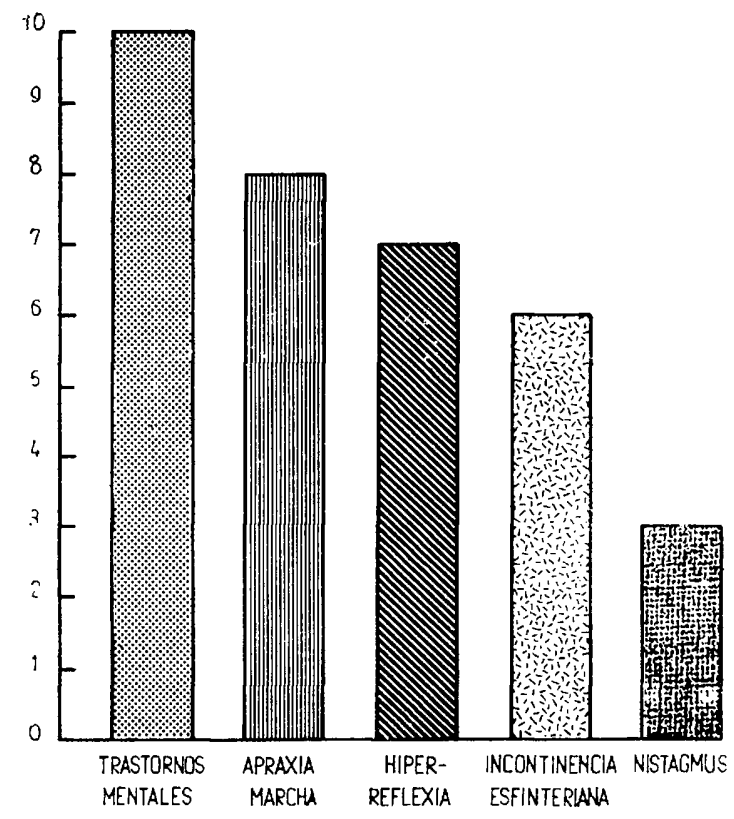

Grifico 1 - Sintomas prominentes en los 10 casos.

Los estudios complementarios en los 11 restantes ro conformaron un diagnóstico definitivamente claro. Por lo tanto, del grupo inicial, 11 pacientes fuerọn excluídos. Esta mención resulta importante por el hecho de que si fué realizado el diagnóstico clínico y complementario de sindrome de hidrocéfalo de presión normal, solamente el $50 \%$ de nuestros pacientes se vió favorecido por la colocación de una válvula de baja presión.

La primera pregunta que nos hacemos es: porqué no mejoraron todos nuestros enfermos, ya que el diagnóstico de hidrocéfalo de baja presión es indudable, por lo menos con los elementos actuales? La respuesta que nos surge es que no todos ellos configuraron la misma entidad clínica. 
La segunda pregunta: si no se trata de la misma enfermedad, que es? Consideramos que el hidrocéfalo de presión normal es un sindrome y que varias causas pueden llevar al mismo hecho clínico. La mejoría observada en aproximadamente el $50 \%$ de los pacientes sería para aquellos en los que se resuelve una causa mecánica. El otro $50 \%$ que ro se ve beneficiado con esta terapéutica, debe ser de enfermedades del grupo degenerativo que no somos capaces de diagnosticar adecuadamente $\mathrm{y}$, por lo tanto, no tienen razón de beneficiarse con el tratamiento quirúrgico.

Una tercera pregunta surge: como diferenciar un grupo del otro? Lamentablemente a la luz de los conocimientos actuales, tanto clínicos como radiológicos, no es posible llegar a esa diferenciación. Se podría sugerir la biopsia cerebral previa a la implantación o no de la válcula; pero todos nosotros conocemos las dificultades de la biopsia cerebral, que no siempre se realiza en un sitio adecuado. Por lo tanto, consideramos en definitiva que lo azaroso de la biopsia cerebral descoloca a ésta como elemento diagnóstico definitorio.

Sin embargo, teniendo en cuenta que, de los enfermos adecuadamente estudiados y diagnosticados que fueron ojerados mejora un 50\%, debemos correr el riesgo y colocar una válvula a todos los que llenan las características clínicas y complementarias de este sindrome, a pesar de que sabemos de antemano que el $50 \%$ de ellos no tendrá beneficio con la operación. Por otra parte, desaconsejamos toda intervención quirúrgica en aquellos enfermos que no reúnen todas las condiciones clinicas y complementarias de este sindrome.

\section{RESUMEN}

Se analizan las condiciones fisiopatológicas del sindrome de hidrocéfalo de presión normal. De un grupo de 21 pacientes con sintomatología clínica correspondiente a esta entidad, se seleccionan 10 que presentan un diagnóstico clínico, radiológico y cisternográfico indudable de la entidad en estudio. Se observó que, a pesar de esta selección solamente un $50 \%$ de ellos se vió beneficiado con la colocación de una válvula de baja presión. Surge entonces la hipotésis de que este cuadro es un sindrome multicausal; que por un proceso fisiopatogenico común produce un cuadro slínico, radiológico y cisternográfico similar y que serian beneficiados únicamente aquel'os enfermos en los que se soluciona el problema mecánico.

\section{SUMMARY}

\section{Considerations on low pressure hydrocephalus}

The physiopathological conditions of the normal pressure hydrocephalus syndrome are analysed. Within a group of 21 patients with clinical sinto- 
mathology pertaining this entity, only were 10 selected with a clinical, radiological and cisternografic indubitable diagnosis. It has been noticed that in spite of the stricked selection only a $50 \%$ of them have been beneficed by the application of a low pressure valve. Then, the hypothesis that the disease is a multicausal syndrome appears, which by an identical physiopathologic process produces or exhibits a similar clinical, radiological and cisternographic picture. Only those patients on whom the mechanical problem is solved would be beneficed by neurosurgery.

\section{REFERENCIAS}

1. ADAMS, R.D.; FISCHER, C.M. \& HAKIM, S. - Symptomatic occult hydrocephalus with normal cerebrospinal fluid pressure. N. England J. Med. 273: 117, 1965.

2. ALMEIDA, G.M.; PEREIRA, W.C.; FACURE, N.O. - Ventrículo-auriculostomia nos bloqueios ao trânsito do liquido cefalorraqueano na cisticercose encefálica. Arq. Neuro-Psiquiat. (São Paulo) 24:163, 1966.

3. BARINI, O. - Hidrocefalo de pressão normal: análise crítica. Seara Médica Neurocirúrgica $1: 261,1973$.

4. BENSON, D.F.; LE MAY, M. \& PATTEN, D.M. - Diagnosis of normal pressure hydrocephalus. N. England J. Med. 283:609, 1970.

5. BRANDEN, J. \& MEYERS, R. - Behaviour of the systemic blood pressure, pulled rate and spinal fluid pressure artiticially produced. Arch. Surg. (Chicago) $36: 1,1938$.

6. BROWN, D.G. \& GOLDENSOHN, E.S. - The electroencephalorgam in normal pressure hydrocephalus. Arch. Neurol. (Chicago) 29:70, 1973.

7. EKBOM, K.; GREITZ, T. \& KUGELBERG, E. - Hydrocephalus due to ectasia of the basilar artery. J. Neurol. Sci. 8:465, 1969.

8. FISHMAN, R.A. — Occult hydrocephalus. New England J. Med. 274:466, 1966.

9. GESCHWIND, N. - The mechanism of normal pressure hydrocephalus. J. Neurol. Sci. $7: 481,1968$.

10. HAKIM, S. - Algunas observaciones sobre la presión del liquido cefalorraquídeo. Sindrome hidrocefálico en el adulto con presión normal del líquído cefalorraquideo. Tesis 957, Universidad Javeriana, Bogotá (Colombia), 1964.

11. HAKIM, S. \& ADAMS, R.D. - The special clinical problem of symptomatic hydrocephalus with normal cerebrospinal fluid pressure. J. Neurol. Sci. 2:307, 1965.

12. HAIKIM, S. - Biomechanics of hydrocephalus. Acta Neurol. Latinoamer. Supl. 1, $17: 169,1971$.

13. HEINZ, E.R.; DAVIS, D.O. \& KARP, H.R. - Abnormal isotope cisternography in symptomatic occult hydrocephalus. Radiology 95:109, 1970.

14. HILL, M.E.; LOUGHEED, W.M. \& BARNETT, H.J.M. - A treatable form of dementia due to normal pressure communicating hydrocephalus. Canad. Med. Assoc. J. 97:1309, 1967 .

15. KIBLER, R.F.; COUCH, R.S.C. \& COMPTON, M.R. - Hydrocephalus in the adult following spontaneous subarachnoid hemmorrage. Brain 84:45, 1961.

16. LE MAY, M. \& NEW, P.F.J. - Radiological diagnosis of occult normal-pressure hydrocephalus. Radiology 96:347, 1970.

17. LEWIN, W. - Preliminary observations on external hydrocephalus after severe head injury. Brit. J. Surg. 55:747, 1969.

18. McCUllough, D.C.; HARBERT, J.C.; DI CHIRO, G. \& OMMAYA, A.K. Prognostic criteria for cerebrospinal fluid shunting from isotope cisternography in communicating hydrocephalus. Neurology (Minneapolis) 20:594, 1970. 
19. MILHOT, T.H.; CLARK, R.G. \& HAMMOCK, M.K. - Gross pathological findings in acute and sub-acute obstructive hidrocephalus in dog and monkey. J. Neurosurg. 32:390, 1970 .

20. NORRELL, H.; WILSON, C.K.; HARRISON, J.; MEAISON, L. \& BERTAN, V. - Venous factors in infantile hâdrocephalus. J. Neurosurg. 31:561, 1969.

21. OJEMANN, R.G. - Normal pressure hydrocephalus. Clin. Neurosurg. 18:337, 1971.

22. OJEMAN, R.G.; FISCHER, C.M.; ADAMS, R.D.; SWEET, W.H. \& NEW, P.J. - Further experience with the syndrome of normal pressure hydrocephalus. J. Neurosurg. 31:279, 1969 .

23. OJEMANN, R.G. - Normal pressure hydrocephalus. In Scientific Foundations of Neurology. William Heinemann Medical Books, London, 1972.

24. RYDER, H.W.; RESENAUR, A.; PENKA, E.J.; ESPEY, F. \& EVANS, J.P. - Failure of abnormal cerebrospinal fluid pressure to influence cerebral function. Arch. Neurol. Psychiat. (Chicago) 70:563, 1953.

25. SCAFF, H.; TSANACLIS, A.M.C. \& SPINA-FRANCA, A. - Hidrocefalo com pressão normal e neurocisticercose. Arq. Neuro-Psiquiat. (São Paulo) 32:223, 1974.

26. SJAASTAD, O. \& NORDVIK, L. - The corpus callosum angle in the diagnosis of cerebral ventricular enlargement. Acta Neurol. Scand. 49:396, 1973.

27. THOMPSON, R.K. \& MALINA, S. - Dinamic axial brain-stem distortion as a mechanism explaining the cardiorespiratory changes in increased intracranial pressure. J. Neurosurg. 16:664, 1959.

28. TSUBOKAWA, T. - The factors of indication on shunt operation for normal pressure hydrocephalus. Neurol. Med. Cir. (Tóquio) 12:316, 1972.

29. WOLINSKY, J.S.; BARNES, B.D. \& MARGOLIS, T. - Diagnostic tests in normal pressure hydrocephalus. Neurology (Minneapolis) 23:706, 1973.

Clínica Neurológica - Hospital Ramos Mejía - Urquiza 609 - Buenos Aires - Argentina. 